\title{
High-Sensitivity Two-Photon Absorption Microcavity Autocorrelator
}

\author{
T. Krug, M. Lynch, A. L. Bradley, J. F. Donegan, L. P. Barry, H. Folliot, J. S. Roberts, and G. Hill
}

\begin{abstract}
A GaAs-AlAs microcavity device has been used as a photodetector in an autocorrelator for measuring the temporal pulsewidth of $1.5-\mu \mathrm{m}$ optical pulses. Enhancement of the two-photon absorption photocurrent due to the microcavity structure results in an autocorrelation (average power times peak power) sensitivity of $9.3 \times 10^{-4}(\mathrm{~mW})^{2}$, which represents two orders of magnitude improvement when compared with conventional autocorrelators.
\end{abstract}

Index Terms-Cavities, correlation, nonlinear detection, optical pulse measurements, photodetectors, photodiodes.

\section{INTRODUCTION}

$\mathbf{T}$ WO-PHOTON absorption (TPA) in semiconductors is an attractive alternative to second-harmonic generation (SHG) for autocorrelation [1], [2] because of lower cost and increased sensitivity. TPA in photodiodes [3], [4] and AlGaAs light-emitting diodes (LEDs) [5] for autocorrelation measurement of picosecond and femtosecond laser pulses has been previously demonstrated. Also, waveguide TPA in commercial laser diodes has been used to fully characterize picosecond pulses in the temporal and phase domain [6]. However, the low efficiency of the TPA process and resulting requirement for high peak powers has to be overcome before it can be satisfactorily exploited in practical optical communications systems. We have recently demonstrated that the TPA photocurrent can be hugely enhanced, by four orders of magnitude, by placing the active material in a microcavity structure [7], [8].

Therefore, in optical telecommunication, as opposed to laser diagnostics, where there is a requirement to measure low peak powers, our $1.5-\mu \mathrm{m}$ TPA microcavity device is an excellent candidate for a detector. The enhancement of the TPA-induced photocurrent due to the cavity finesse greatly improves the sensitivity of the autocorrelation measurement. The growth of these devices will also be significantly cheaper due to the vertical device orientation compared with waveguide TPA structures. Furthermore, the vertical nature and relatively large area of the structure allows for easier coupling than waveguide

Manuscript received November 12, 2003; revised January 13, 2004 This work was supported in part by Enterprise Ireland under Contract ATRP/2002/301 B.

T. Krug, M. Lynch, A. L. Bradley, and J. F. Donegan are with the Semiconductor Photonics Group, Physics Department, Trinity College Dublin, University of Dublin, Dublin 2, Ireland (e-mail: krugt@tcd.ie).

L. P. Barry is with the Department of Electronic Engineering, Dublin City University, Dublin 9, Ireland.

H. Folliot is with the Laboratoire de Physique des Solides, INSA, Rennes Cedex 35043, France.

J. S. Roberts and G. Hill are with the Department of Electronic and Electrical Engineering, University of Sheffield, Sheffield S1 3JD, U.K.

Digital Object Identifier 10.1109/LPT.2004.827102

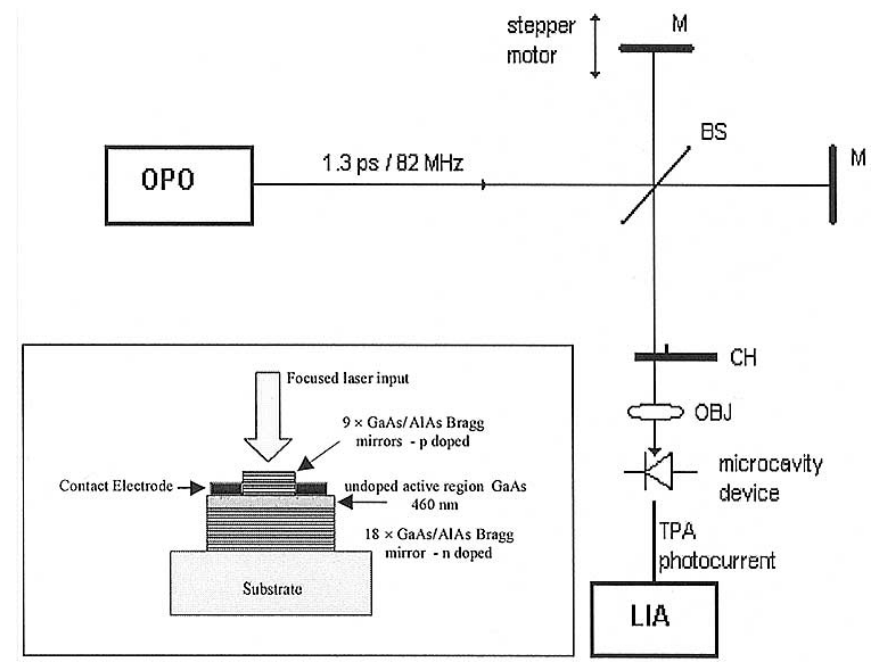

Fig. 1. Experimental setup of autocorrelation measurement. BS: beam splitter. $\mathrm{CH}$ : chopper. $\mathrm{M}$ : mirror. LIA: lock-in amplifier. Inset: schematic of microcavity device structure.

structures. In addition, the fact that the device is thin means there are no phase matching problems compared with SHG crystals and free-space optics. In this letter, we measured the sensitivity of TPA microcavity devices in an autocorrelation configuration and compare with other autocorrelator sensitivities from the literature.

\section{Device Structure}

The TPA microcavity consists of two distributed Bragg reflector (DBR) mirrors surrounding an undoped GaAs active region (inset Fig. 1). The active region material has a bandgap energy of $1.428 \mathrm{eV}$. The thickness of the active region is $460 \mathrm{~nm}$. There are 18 periods in the n-type bottom mirror and 9 periods in the p-type top mirror. The mirrors consist of alternating 134.3-nm AlAs and 115.7-nm GaAs layers. The p- and n-DBR mirrors are doped to a concentration $>10^{17} \mathrm{~cm}^{-3}$ with $\mathrm{C}$ and $\mathrm{Si}$, respectively.

The DBR stopband extends from 1475 to $1630 \mathrm{~nm}$ with a reflectivity resonance minimum at $1521 \mathrm{~nm}$, the linewidth of the cavity resonance is $4 \mathrm{~nm}$ (Fig. 2). The DBR mirror reflectivities for the front and back mirrors are $R=95 \%$ and $R=98.6 \%$, respectively, and the cavity finesse $F$ is 96 . Previously, we have shown the dependence of the device efficiency on the cavity finesse and pulsewidth [7]. This device has been especially designed for use with pulses at a wavelength of $1.5 \mu \mathrm{m}$, with 1-ps temporal pulsewidth. The match of the microcavity spectral bandwidth to that of the incoming pulse can be seen in 


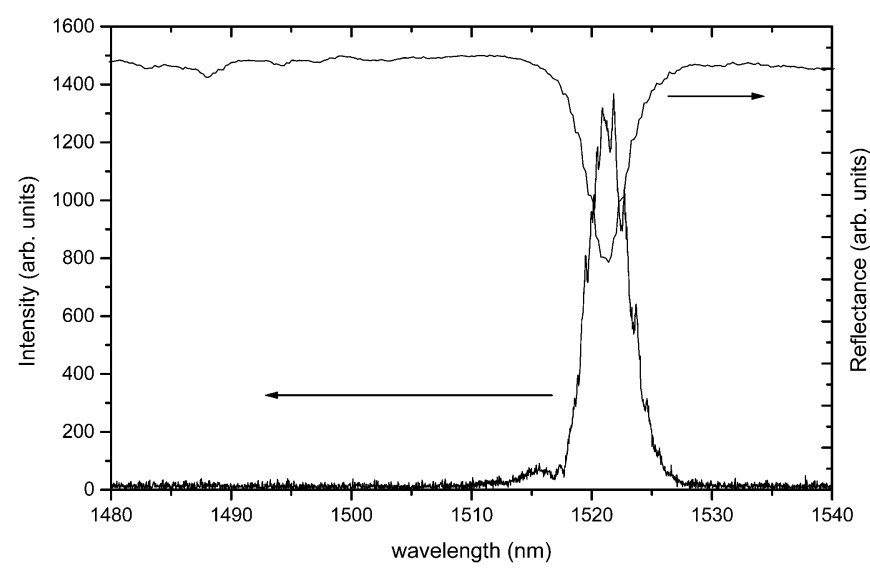

Fig. 2. Reflectivity spectrum of the microcavity device and pulse spectrum.

Fig. 2. For shorter and longer pulsewidths, it is possible to design the microcavity mirrors to match the cavity lifetime to the pulsewidth and, thereby, to produce the maximum enhancement of the TPA.

\section{EXPERIMENT}

The noninterferometric autocorrelation is measured using a standard Michelson interferometer configuration. The second harmonic crystal followed by a highly sensitive photodetector used in the conventional schemes was simply replaced by the TPA microcavity photodetector. The experimental setup is shown in Fig. 1. The optical pulses were generated by an optical parametric oscillator (OPO) synchronously pumped by a Ti : Sapphire laser system, at a repetition rate of $82 \mathrm{MHz}$. At a wavelength of $1.5 \mu \mathrm{m}$, the typical temporal pulsewidth was 1.3 ps. After traversing the two arms of the interferometer, the two beams are colinearly focused to a $12-\mu \mathrm{m}$ diameter spot on the microcavity device using a $\times 10$ microscope objective (OBJ). The TPA photocurrent was measured using a lock-in technique. The best sensitivity is achieved using the shunt resistance $R_{\text {SHUNT }}=1 M \Omega$ of the microcavity device as the load resistor.

The sensitivity of the TPA microcavity autocorrelator was measured by inserting neutral density filters in the beam path to control the signal-to-noise ratio (SNR) levels. Fig. 3 shows the microcavity device photocurrent as a function of delay for an incident average power of $0.77 \mu \mathrm{W}$ and peak power of $3.6 \mathrm{~mW}$. The quadratic response of the TPA photocurrent (versus incident average power) of the devices was verified down to $1 \mu \mathrm{W}$. The measured slope of the response at $1 \mu \mathrm{W}$ was 2.4. This value is greater than the expected value of 2.0 and may be related to defect levels in the active region. Devices with an active region around $890 \mathrm{~nm}$ show a slope efficiency of close to 2.0 as expected [7]. Given the nonlinear response of the photocurrent with incident power, it is possible to extrapolate the incident power to an SNR of 1 [9]. The sensitivity of the autocorrelator, defined as the product of the peak and average power of the minimum detectable signal $(\mathrm{SNR}=1)$, is found to be $9.3 \times 10^{-4}(\mathrm{~mW})^{2}$ at a bandwidth of $1 \mathrm{~Hz}$, where the bandwidth of $1 \mathrm{~Hz}$ is the bandwidth of the lock-in amplifier.

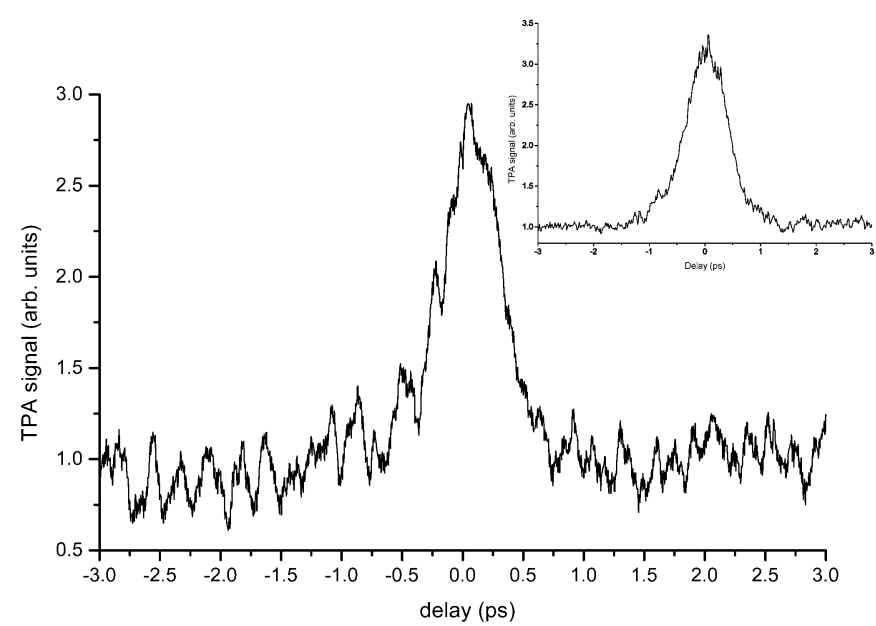

Fig. 3. Intensity autocorrelation of the laser pulse obtained with a Michelson interferometer and a TPA microcavity photodetector for average incident power of $0.77 \mu \mathrm{W}$. Inset: intensity autocorrelation trace obtained at higher average incident power $(\sim 100 \mu \mathrm{W})$.

For a given shunt resistance $R_{\mathrm{SHUNT}}$, the limit for the detectable photocurrent is governed by the thermal noise $I_{\mathrm{th}}^{2}=$ $4 k T B_{N} / R_{\mathrm{SHUNT}}$, where $k$ is the Boltzmann constant, $T$ the temperature, and $B_{N}=1.6 \mathrm{~Hz}$ the measurement bandwidth. Comparing the theoretical value of the thermal noise $I_{\mathrm{th}}=$ $0.16 \mathrm{pA}$ with the standard deviation of the data in the side arms of the autocorrelation trace in Fig. $3, I_{\text {th }}=0.1 \mathrm{pA}$, we observe good agreement. The shunt resistor of the device is measured to be $1 M \Omega$ and, because of the smaller area of the device, is less than that achievable with a typical waveguide device.

To show the enhancement in sensitivity due to the microcavity, the autocorrelation measurement has also been performed at a wavelength of $1.46 \mu \mathrm{m}$ off the stopband of the reflectivity spectrum microcavity's DBRs. The magnitude of photocurrent measured off-band $I_{\mathrm{TPA}}=220 \mathrm{pA}$ is consistent with the theoretical values obtained by assuming TPA in bulk material with the same thickness as the active region of the microcavity device, given a TPA coefficient of $\beta=3 \times 10^{-10} \mathrm{~m} / \mathrm{W}$. A sensitivity of $1.5 \times 10^{3}(\mathrm{~mW})^{2}$ is determined, corresponding to an average power of $330 \mu \mathrm{W}$ and peak power of $1.5 \mathrm{~W}$. The sensitivity of the TPA microcavity autocorrelation measurements presented here compares very favorably with conventional autocorrelators based on SHG techniques, which typically have a sensitivity of $1(\mathrm{~mW})^{2}$ [10]. The sensitivity value is also higher than those reported using a silicon waveguide TPA autocorrelator at $1.5 \mu \mathrm{m}, 1(\mathrm{~mW})^{2}$ [11], and a silicon avalanche photodiode [12]. Compared to commercially available GaAsP photodiodes, AlGaAs LEDs and GaAs LEDs recently used for TPA autocorrelation at $1.5 \mu \mathrm{m}$, the microcavity device is found to be more sensitive by at least a factor of ten [13] at a bandwidth of $1 \mathrm{~Hz}$. However, waveguide devices and photomultiplier tubes are still more sensitive due to longer active regions, especially when used in direct-detection photon counting mode. For an InGaAsP diode, a sensitivity of $\sim 0.15 \times 10^{-3}(\mathrm{~mW})^{2}$ was stated [13], and for a GaAs photomultiplier tube, a peak times average power sensitivity of $1.7 \times 10^{-4}(\mathrm{~mW})^{2}$ at $1.5 \mu \mathrm{m}$ was reported [14]. 


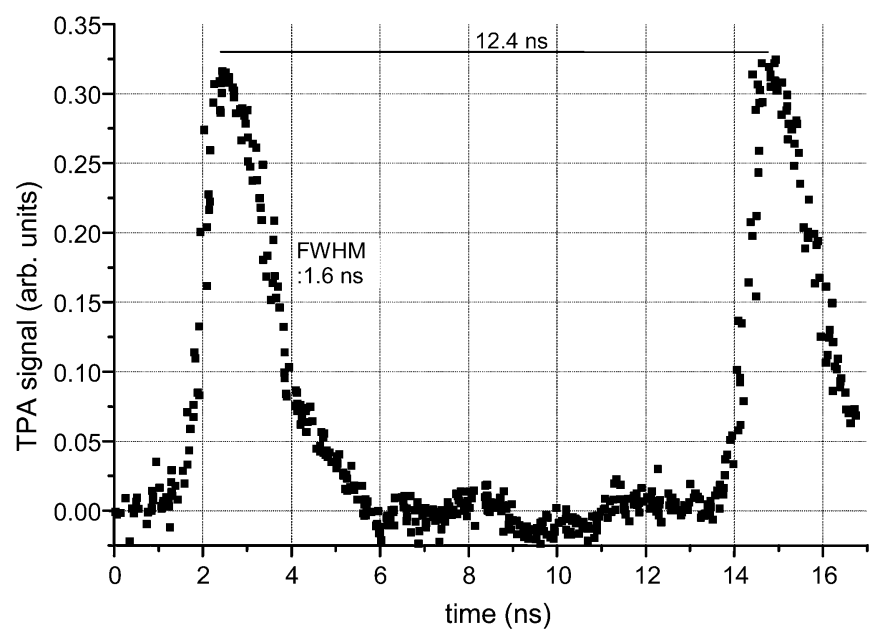

Fig. 4. Electrical response of the TPA microcavity device to incident $1.5-\mu \mathrm{m}$ pulses at a repetition rate of $82 \mathrm{MHz}$ in unbiased configuration. Bandwidth $=$ $550 \mathrm{MHz}$.

Microcavity enhanced TPA photodetectors should also find use in optical time-division multiplexing systems. A key issue for demultiplexing is the device response, determining the time interval required between sampling two data bits. The TPA microcavity device response was measured using a sampling scope to detect the TPA generated photocurrent as a function of time.

Fig. 4 shows the measured electrical response for an unbiased device, with an incident average power of $8.5 \mathrm{~mW}$, at $1.5 \mu \mathrm{m}$ and 1.3-ps pulsewidth. By measuring the decay time between $90 \%$ and $10 \%$ of the peak value, an approximate bandwidth of $550 \mathrm{MHz}$ can be extracted. These proof-of-concept devices have not been impedance matched or optimized for electrical response and, therefore, significantly higher bandwidths can be expected.

\section{CONCLUSION}

We have used microcavity enhanced TPA in a GaAs-AlAs photodetector for high sensitivity autocorrelation measurements, suitable for low-power $1.55-\mu \mathrm{m}$ wavelength optical pulses. A sensitivity of $9.3 \times 10^{-4}(\mathrm{~mW})^{2}$ at $1 \mathrm{~Hz}$ is reported and represents an improvement by two orders of magnitude when compared with commercially available SHG devices.

\section{REFERENCES}

[1] H. K. Tsang, L. Y. Chan, J. B. D. Soole, H. P. LeBlanc, M. A. Koza, and R. Bhat, "High sensitivity autocorrelation using two-photon absorption in InGaAsp waveguides," Electron. Lett., vol. 31, no. 20, pp. 1773-1775, 1995.

[2] Y. Takagi, "Simple autocorrelator for ultraviolet pulse width measurements based on the nonlinear photoelectric effect," Appl. Opt., vol. 33, no. 27, pp. 6328-6332, 1994.

[3] Y. Takagi, T. Kobayashi, K. Yoshihara, and S. Imamura, "Multiple- and single-shot autocorrelator based on two-photon conductivity in semiconductors," Opt. Lett., vol. 17, no. 9, pp. 658-660, 1992.

[4] J. K. Ranka, A. L. Gaeta, A. Baltuska, M. S. Pshenichnikov, and D. A. Wiersma, "Autocorrelation measurement of 6-fs pulses based on the two-photon-induced photocurrent in a GaAsp photodiode," Opt. Lett., vol. 22, no. 17, pp. 1344-1346, 1997.

[5] D. T. Reid, M. Padgett, C. McGowan, W. E. Sleat, and W. Sibbett, "Light-emitting diodes as measurement devices for femtosecond laser pulses," Opt. Lett., vol. 22, no. 4, pp. 233-235, 1997.

[6] D. T. Reid, B. C. Thomson, J. M. Dudley, and J. D. Harvey, "Sonogram characterization of picosecond pulses at $1.5 \mu \mathrm{m}$ using waveguide two photon absorption," Electron. Lett., vol. 36, no. 13, pp. 1141-1142, 2000.

[7] H. Folliot, M. Lynch, L. P. Barry, A. L. Bradley, L. A. Dunbar, J. Hegarty, J. F. Donegan, J. S. Roberts, and G. Hill, "Two-photon absorption photocurrent enhancement in bulk AlGaAs semiconductor microcavities," Appl. Phys. Lett., vol. 80, pp. 1328-1330, 2002.

[8] H. Folliot, M. Lynch, A. L. Bradley, T. Krug, L. A. Dunbar, J. F. Donegan, and L. P. Barry, "Two-photon-induced photoconductivity enhancement in semiconductor microcavities: A theoretical investigation," J. Opt. Soc. Amer. B, vol. 19, no. 10, pp. 2396-2402, 2002.

[9] T. Krug, M. Lynch, A. L. Bradley, and J. F. Donegan, "Two-photon absorption in microcavities for optical autocorrelation and sampling," in Tech. Proc. Conf. Lasers and Electro-Optics (CLEO)/Eur. Quantum Electronics Conf. (EQEC), Munich, Germany, 2003, Paper CE5-4-Thu.

[10] Autocorrelator PulseScope Manual, Angewandte Physik \& Elektronik GmbH, APE, Apr. 2001, p. 9.

[11] T. K. Liang, H. K. Tsang, I. E. Day, J. Drake, A. P. Knights, and M. Asghari, "Silicon waveguide two-photon absorption detector at $1.5 \mu \mathrm{m}$ wavelength for autocorrelation measurements," Appl. Phys. Lett., vol. 81, no. 7, pp. 1323-1325, 2002.

[12] K. Kikuchi, "Optical sampling system at $1.5 \mu \mathrm{m}$ using two photon absorption in Si avalanche photodiode," Electron. Lett., vol. 34, no. 13, pp. 1354-1355, 1998.

[13] L. P. Barry, B. C. Thomson, J. M. Dudley, and J. D. Harvey, "Autocorrelation and ultrafast optical thresholding at $1.5 \mu \mathrm{m}$ using a commercial InGaAsp $1.3 \mu \mathrm{m}$ laser diode," Electron. Lett., vol. 34, no. 4, pp. 358-360, 1998.

[14] J. M. Roth, T. E. Murphy, and C. Xu, "Ultrasensitive and high-dynamicrange two-photon absorption in a gaas photomultiplier tube," Opt. Lett., vol. 27 , no. 23, pp. 2076-2078, 2002. 